\title{
Nanofibre network rooted from the alkaline peroxide treatment of oil palm empty fruit bunches
}

\begin{abstract}
The increasing popularity of the oil palm empty fruit bunches (EFB) as a source of non-wood fibre has prompted a variety of research on processing and utilisation of the material. In an attempt to define the characters, reusability and end-of-life, oil palm EFB was processed by the alkaline peroxide variable treatment (APVT) systems. Low synergy between alkaline peroxide (AP) chemical and mechanical fibrillation through fibrillation (CMR synergy) revealed the yield of segments of EFB vascular bundles while heightening the mechanical forces further, generated more uniform but a mixture of fiber and segments of fibre bundles. An intermediate CMR synergy generated fibres forming a more well-defined but a rough resultant fibre network due to partial fibrillation of the vascular bundle. Applying maximal CMR synergy was found to generate higher yield of network strengthening fibrous cells. These were later identified as nanoscale fiber network or nanoscan, consisting of 10-80 nm diameter fibers arranging themselves in a systematic network. Analysis of the polarity of fibers harvested from the APVT systems manifests the systematic construction of nanofibrils winding in helical manner to form arrays of nanofibres that glue themselves together as micro-fibrils. Interconnections between fibers and other gluing elements led to the vascular bundle known as the EFB biomass that was once dross and that can now be marvelled as an alternative source of nanofibers for the nanoindustry sector.
\end{abstract}

Keyword: Biomass; EFB; Nano-elements; Nano-fibers 